\title{
Models matter in metastasis
}

\begin{abstract}
A xenograft is a transplant that occurs between two different species. A healthy immune system will predictably reject such inter-species transplants, necessitating the use of immunodeficient animals. The athymic nude mouse, an early model developed in the 1960s, remains a staple among researchers studying human cancers in animal models. However, more recently developed models with even greater immune impairments may offer improved tumor acceptance and growth. An increasingly popular example is the NSG model, a NOD-SCID mouse with deleted IL2 $\gamma$ receptors.

Although immunodeficient mice will bear transplanted tumors, recapitulating metastasis is trickier. In breast cancer, metastasis is predictable in humans but limited in nude mice. Some spreading will occur as expected, but metastases in the liver, bones, or the brain are generally absent, impeding research into the laterand more lethal-stages of the disease. Looking for spontaneous metastasis to the brain, Jennifer Koblinski and her colleagues turned to the NSG mouse. Their nude-to-
\end{abstract}

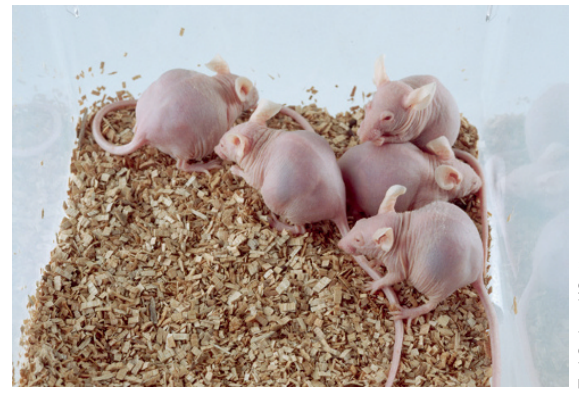

NSG comparison can be found in PLoS One (11, e0163521; 2016).

The team tested spontaneous metastasis using three breast cancer lines xenografted into the mammary glands of the mice. The results were striking. According to Koblinski, "some of the tumor cell lines barely grow in the nude mice but exhibit significant growth in the NSG." NSG mice also developed metastases in more organs, including the brain, and at greater ratesup to $100 \%$ for some lines. Experimental metastasis from direct intracardiac injections confirmed similar results.

Convinced this is a good model for her research, Koblinski commented, "The NSG model will allow me to look at breast cancer metastasis to the brain using a spontaneous model as well as an experimental model." Although she agrees that the move towards immunocompetent models is important to understand the immune system's role in cancer, transgenic mice just don't exhibit the same consistent patterns of metastasis. Allograft models, in which transplants occur between the same strains of mice with normal immune systems, are promising, but there is currently only one-the 4T1 mouse mammary tumor cell line-available.

Koblinski, who leads the Cancer Mouse Models Core at the VCU Massey Cancer Center, hopes their work will encourage others to consider the NSG mouse. She notes that researchers studying other cancers at her facility are also observing differences in tumor growth and metastasis in NSG mice compared to what is reported in the literature using nude models. "We're finding that NSG mice are just better models for studying tumor growth and metastasis of various cancers, as well as for treatment studies with different drugs."

Ellen P. Neff

\section{WAKING UP WITH DOPAMINE}

Despite the terminology (being "put to sleep" and "waking up"), the effects of anesthesia more closely resemble that of a coma rather than natural sleep_-patients are profoundly unresponsive and lack basic reflexes. The drugs used to put a patient to sleep are carefully administered and monitored during a procedure, but waking a patient back up is a much more passive process. Afterwards, the patient simply remains unconscious until their body eliminates the anesthetic used. The time that it takes to achieve wakefulness can vary, which raises the risks of respiratory complications the longer a patient requires artificial ventilation. Upon waking, confusion and grogginess often linger.

The ability to actively wake a patient from general anesthesia could save time in the recovery room, minimize respiratory risks, and possibly help reduce the subsequent mental fog many patients experience. Dopamine-a neurotransmitter associated with wakefulness-is emerging as potential target for the task. A team based in Boston has previously demonstrated that drugs commonly used to treat ADHD, which act upon dopamine in the brain, are capable of arousing rats under continuous steady-state general anesthesia induced by three separate drugs: isoflurane, propofol, and sevoflurane. Now, they are turning their attention to the underlying neural circuitry behind this effect, using optogenetic techniques to specifically test the role of dopamine neurons in waking from anesthesia (Proc. Natl. Acad. Sci. USA 113, 12826-12831; 2016).

The team surgically implanted small optical fibers into the brains of DAT-cre mice, targeting the dopamine receptor-rich ventral tegmental area (VTA). An experimental group of mice was also injected with FLEX-ChR2, a fluorescent channelrhodopsin expressed in dopamine receptors. Dopamine receptors in ChR2+ mice can then be specifically activated using light from the implanted optical fibers.

Upon optical stimulation, all six ChR2+ mice righted themselves, despite continuous administration of isoflurane, while those without the gene remained on their backs. ChR2+ mice whose dopamine receptors were blocked with a D1 agonist also remained unconscious. The righting reflex is an indication of arousal from the coma-like anesthetic state, as rodents will exhibit this response even while sleeping. Further, EEGs of the ChR2+ mice looked similar to those of conscious animals. These results confirm the team's previous hypothesis that dopamine and its associated receptors are actively involved with regaining consciousness from general anesthesia. 